\title{
Susceptibility of Neisseria gonorrhoeae to cefotaxime and ceftizoxime
}

\author{
E H SNG, $†$ V S RAJAN, ROGER PANG, AND K L YEO† \\ From the $†$ Department of Pathology, Outram Road, Singapore, and $¥$ Middle Road Hospital, Singapore
}

SUMMARY Two hundred and thirty-nine penicillinase-positive and 240 penicillinase-negative strains of Neisseria gonorrhoeae were tested for their susceptibility to cefotaxime. All were susceptible to cefotaxime at a concentration of $0.062 \mu \mathrm{g} / \mathrm{ml}$. Penicillinase-negative strains were less susceptible than penicillinase-positive strains. This is attributed to the use of prophylactic antibiotics by prostitutes, which has led to the selection of less susceptible penicillinase-negative strains but has not affected the susceptibility of penicillinase-positive strains. The minimum inhibitory concentrations of cefotaxime showed significant positive correlations with those of penicillin, ampicillin, tetracycline, and kanamycin but had a negative correlation with spectinomycin. The related cephalosporin, ceftizoxime, was found to be as effective as cefotaxime against penicillinase-positive and penicillinase-negative gonococci.

\section{Introduction}

Cefotaxime is a new cephalosporin that is highly effective against a wide range of bacteria ${ }^{2}$ and against penicillinase-positive (PPNG) and penicillinase-negative (non-PPNG) strains of Neisseria gonorrhoeae. ${ }^{34}$ Its level of activity against the gonococcus is several times greater than that of other cephalosporins (cefuroxime) and cephamycins (cefoxitin), which are currently used in the treatment of gonorrhoea caused by PPNG. In view of its potential value as a second-line antibiotic we have recently started monitoring the activity of this drug against routine isolates of gonococci. This paper reports its activity against 479 strains of gonococci and discusses the variation in the susceptibility of the gonococci isolated from different sources.

Ceftizoxime is another cephalosporin which has been recently introduced. It is closely related to cefotaxime, to which its antibacterial activity is comparable. ${ }^{5}$ We have tested 72 strains of gonococci for their susceptibility to ceftizoxime to determine if its activity against the gonococcus is comparable to cefotaxime.

\section{Materials and methods}

The 479 strains of $N$ gonorrhoeae were recent isolates from routine specimens. Isolates from the ano-

Address for reprints: Dr E H Sng, Department of Pathology, Outram Road, Singapore 0316, Republic of Singapore

Accepted for publication 13 October 1980 genital region were identified by their colonial $œ$ morphology on modified Thayer-Martin medium, oxidase reaction, and Gram-stained microscopical appearance; isolates from other sites were further confirmed by sugar utilisation tests on cystinetrypticase agar. All penicillinase-producing strains were confirmed by the rapid iodometric method. ${ }^{6}$

ANTIBIOTIC SUSCEPTIBILITY

The minimum inhibitory concentrations (MICs) of cefotaxime and ceftizoxime were determined by the agar-plate dilution method. Two-fold dilutions of the antibiotics from $0.125 \mu \mathrm{g} / \mathrm{ml}$ to $0.0005 \mu \mathrm{g} / \mathrm{ml}$ were $\frac{5}{3}$ prepared in GC agar base (BBL) supplemented with $1 \%$ haemoglobin (BBL) and IsoVitalex.

Strains of $N$ gonorrhoeae were grown overnight on chocolate agar. A suspension of the organism in $ᄋ$ trypticase soy broth was made and the turbidity $D$ adjusted to correspond to that of $0.5 \%$ McFarland standard barium sulphate solution. The suspension N was further diluted $1 / 100$ to give a final cell count of approximately $10^{6}$ organisms per $\mathrm{ml}$. Approximately $5-\mu$ l volumes of each suspension were then spotted on $\omega$ the series of plates by a multipoint inoculator. The plates were dried and incubated in $\mathrm{CO}_{2}$ tins at $35^{\circ} \mathrm{Co}$ overnight. The MIC was read as the lowest concen- $\mathbb{\Phi}$ tration of antibiotic that permitted the growth of no $\stackrel{?}{?}$ more than one colony.

To determine the correlations between the MICs of the various antibiotics 82 strains were tested for their $\stackrel{\square}{\square}$ susceptibility to cefotaxime, penicillin, ampicillin, $\stackrel{\varnothing}{\unrhd}$ tetracycline, spectinomycin, and kanamycin. The 
dilution for each antibiotic was such that growth occurred at the lower end of the range.

\section{TESTS OF SIGNIFICANCE}

The MIC distributions of cefotaxime for the different groups of $\mathrm{N}$ gonorrhoeae were compared by the $\chi^{2}$ test. The product moment correlation coefficient ( $r$ ) was used to determine the relationships between the MICs of the various antibiotics. Student's $t$ test was used to compare the geometric means of the MICs of penicillin for non-PPNG from men and women.

\section{Results}

Two hundred and thirty-nine PPNG and 240 nonPPNG strains were examined for their susceptibility to cefotaxime. All the strains were susceptible to $0.062 \mu \mathrm{g} / \mathrm{ml}$. The non-PPNG were less susceptible than the PPNG; $19.6 \%$ of non-PPNG had MIC values of $\geqslant 0.016 \mu \mathrm{g} / \mathrm{ml}$ compared with 5\% of PPNG (table I). The distributions of their MICs are significantly different $\left(\chi^{2}=36 \cdot 9092 ; \mathrm{P}<0 \cdot 001\right)$.

The least susceptible strains were the non-PPNG isolated from women, $23.7 \%$ of which had MICs of $\geqslant 0.016 \mu \mathrm{g} / \mathrm{ml}$. The most susceptible strains were PPNG from men and women, of which $6.5 \%$ and $4 \cdot 1 \%$ respectively had MICs of $\geqslant 0 \cdot 016 \mu \mathrm{g} / \mathrm{ml}$. The MIC distribution of non-PPNG from women is significantly different from those of PPNG isolated from either men or women (table II).

In contrast to isolates from women, only $9.9 \%$ of non-PPNG from men had MICs of $\geqslant 0.016 \mu \mathrm{g} / \mathrm{ml}$, and the MIC distribution of these strains did not differ significantly from those of non-PPNG from women or PPNG from men and women (table II).

MICs of cefotaxime showed strong positive correlation with those of penicillin. Both antibiotics showed significant positive correlations with ampicillin, tetracycline, and kanamycin, but had negative correlations with spectinomycin (table III).

To determine if the MICs of penicillin for nonPPNG strains from men and women were different,
TABLE II Comparison of MIC distributions of cefotaxime for $N$ gonorrhoeae from men $(M)$ and women $(F)$

\begin{tabular}{llcl}
\hline Isolates & Isolates & $\chi^{2}$ test & Probability \\
\hline Non-PPNG (F) & PPNG (F) & 36.4815 & $<0.001$ \\
Non-PPNG (F) & PPNG (M) & 20.3809 & $<0.01$ \\
Non-PPNG (F) & Non-PPNG (M) & 14.0656 & $<0.1$ \\
Non-PPNG (M) & PPNG (F) & 11.2665 & $>0.1$ \\
Non-PPNG (M) & PPNG (M) & 9.8131 & $>0.1$ \\
PPNG (M) & PPNG (F) & 4.6681 & $>0.5$ \\
\hline
\end{tabular}

TABLE III Correlations between MICs of cefotaxime and penicillin with MICs of other antibiotics

\begin{tabular}{|c|c|c|c|c|}
\hline \multirow[b]{2}{*}{ Antibiotics } & \multicolumn{2}{|c|}{ Cefotaxime $(\mu \mathrm{g} / \mathrm{ml})$} & \multicolumn{2}{|c|}{ Penicillin $(\mu \mathrm{g} / \mathrm{ml})$} \\
\hline & $r$ & $P$ & $r$ & $P$ \\
\hline Penicillin & 0.6636 & $<0.001$ & & \\
\hline Ampicillin & 0.6039 & $<0.001$ & 0.8797 & $<0.001$ \\
\hline Tetracycline & $0 \cdot 5267$ & $<0.001$ & $0 \cdot 7211$ & $<0.001$ \\
\hline Spectinomycin & -0.0304 & $>0.05$ & -0.1374 & $>0.1$ \\
\hline Kanamycin & $0 \cdot 3934$ & $<0.001$ & $0 \cdot 2403$ & $<0.05$ \\
\hline
\end{tabular}

$\mathrm{r}=$ product moment correlation coefficient

$\mathrm{P}=$ probability

as was the case with cefotaxime, the susceptibility of 253 strains to penicillin was investigated. It was found that strains from women were more resistant than those from men (table IV). The geometric mean MIC of isolates from women was $2 \cdot 14 \mu \mathrm{g} / \mathrm{ml}$ compared with $0.77 \mu \mathrm{g} / \mathrm{ml}$ for strains from men. The difference was significant $(t=2 \cdot 6136 ; \mathrm{P}<0 \cdot 01)$.

TABLE IV MICs of penicillin for $N$ gonorrhoeae from men and women

\begin{tabular}{lcc}
\hline$\mu g / m l$ & Men & Women \\
\hline$\leqslant 0 \cdot 016$ & 1 & \\
$0 \cdot 031$ & 13 & 4 \\
$0 \cdot 062$ & 26 & 10 \\
$0 \cdot 125$ & 22 & 13 \\
$0 \cdot 25$ & 29 & 15 \\
$0 \cdot 5$ & 27 & 11 \\
$1 \cdot 0$ & 21 & 21 \\
$2 \cdot 0$ & 19 & 16 \\
$4 \cdot 0$ & 1 & 3 \\
$8 \cdot 0$ & 159 & 1 \\
Total & 1 & 94 \\
\hline
\end{tabular}

TABLE I MICs of cefotaxime for Neisseria gonorrhoeae

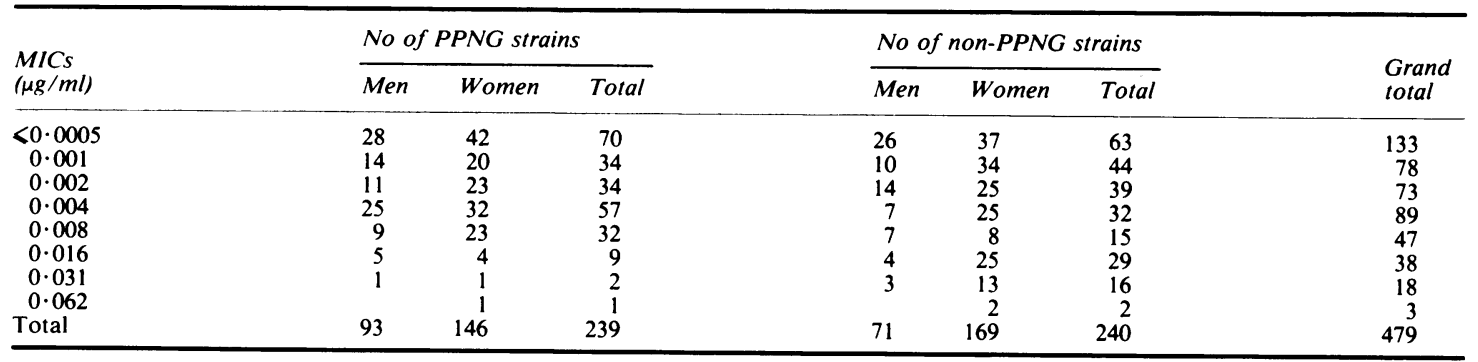


TABLE V MICs of ceftizoxime for $N$ gonorrhoeae

\begin{tabular}{llc}
\hline$\mu g / m l$ & $P P N G$ & Non-PPNG \\
\hline$\leqslant 0.0005$ & 16 & 18 \\
$0 \cdot 001$ & 9 & 5 \\
$0 \cdot 002$ & 9 & 7 \\
$0 \cdot 004$ & 4 & 2 \\
$0 \cdot 008$ & 38 & 2 \\
Total & 38 \\
\hline
\end{tabular}

Seventy-two strains were also tested for their susceptibility to ceftizoxime. All the strains were susceptible to $0.008 \mu \mathrm{g} / \mathrm{ml}$ (table V).

\section{Discussion}

These findings confirm previous studies ${ }^{34}$ that cefotaxime is highly effective against PPNG and non-PPNG strains. All the strains tested were susceptible to a concentration of $0.062 \mu \mathrm{g} / \mathrm{ml}$.

The MICs for non-PPNG are significantly higher than those for PPNG strains. This differs from our previous study, ${ }^{3}$ in which it was found that PPNG strains were less susceptible to cefotaxime than nonPPNG. This is probably due to the difference in the types of patients from whom the gonococci were isolated. Non-PPNG from women had higher MICs than non-PPNG from men or PPNG from men or women, and non-PPNG from women had higher MICs of penicillin than similar strains from men. Since there was a significant positive correlation between MICs of cefotaxime and penicillin, any factor which produces a higher MIC of penicillin for strains from women is also likely to have contributed to a higher MIC of cefotaxime for such strains.

Almost all the specimens from women in the present study came from prostitutes, but only about $10 \%$ of those from men were from male prostitutes. A study presently being conducted shows that over $20 \%$ of male and female prostitutes are receiving prophylactic antibiotics at any one time. It is likely that the use of prophylactic antibiotics had resulted in a selection of strains which were less susceptible to penicillin among the prostitutes. Since almost all the women were prostitutes compared with only $10 \%$ of the men, it is not surprising that the MIC of penicillin for non-PPNG strains from women were higher than those from men. Because of the positive correlation between MICs of penicillin and cefotaxime, the latter for non-PPNG strains from women were also higher than those from men.

The use of prophylactic antibiotics has apparently not resulted in any selective pressure in the case of PPNG. Hence no difference is observed in the MIC distributions of PPNG from men and women. Such strains were much more susceptible than non-PPNG from women. As only $10 \%$ of men were prostitutes, the MICs of non-PPNG from men were only slightly higher than the PPNG from men and women.

This study shows that the taking of prophylactic? antibiotics may be an important factor in causing differences in the antibiotic susceptibility of gonococci from different populations. The antibiotic $\frac{}{\circ}$ taken need not necessarily be the same as the one $\frac{\bar{\sigma}}{2}$ being tested, provided the two drugs show a strong $\widetilde{\Phi}$ positive correlation.

The correlation of cefotaxime to other antibiotics ${ }^{\text {के }}$ is very similar to that of penicillin. Both showed $\overrightarrow{0}$ significant positive correlation to ampicillin, $\vec{\overrightarrow{ }}$ tetracycline, and kanamycin and a negative correla- $\omega$ tion to spectinomycin. This suggests that cefotaxime may be a suitable alternative to spectinomycin or vice ${ }^{-}$. versa in the event of treatment failure with either ${ }_{\omega}^{.}$ drug.

Ceftizoxime has a chemical structure very similar $\vec{N}$ to that of cefotaxime ${ }^{5}$ and a similar range ofo antibacterial activity. This study shows that it is at $\rightarrow$ least as effective against PPNG and non-PPNGC strains as cefotaxime. Like cefotaxime it is also stable to hydrolysis by a variety of $\beta$-lactamases. ${ }^{7}$ Cefotaxime, in a dose of $500 \mathrm{mg}$, has been used $\infty$ successfully in the treatment of gonorrhoea. ${ }^{8}$ Thus, it ${ }^{.}$ is likely that ceftizoxime will also prove to be a potent drug in the treatment of gonorrhoea.

We thank Hoechst, Germany, and Fujisawa Pharmaceutical Co, Japan, for the supply of cefotaxime and ceftizoxime respectively.

\section{References}

1. Sosna JP, Murray PR, Medoff G. Comparison of the in-vitro activities of HR 756 with cephalothin, cefoxitin, and cefaman-: dole. Antimicrob Agents Chemother 1978; 14:876-9.

2. Wise R, Rollason T, Logan M, Andrews JM, Bedford KA. HR 756, a highly active cephalosporin: comparison with cefazolin and carbenicillin. Antimicrob Agents Chemother 3 1978; 14:807-11.

3. Tan RJS, Sng EH, Rajan VS, Lim AL, Yeo KL, Lim EW. O Evaluation of cefotaxime (HR 756) - a new cephalosporin against penicillinase-producing strains of Neisseria gonor-O rhoeae. Asian J Infect Dis 1978; 2:239-41.

4. Baker $C N$, Thornsberry $C$, Jones RN. In-vitro antimicrobial $>$ activity of cefoperazone, cefotaxime, moxalactam (LYO 127935), azlocillin, mezlocillin and other $\beta$-lactam antibiotics against Neisseria gonorrhoeae and Haemophilus influenzae, $\mathrm{N}$ including $\beta$-lactamase-producing strains. Antimicrob Agents $\mathrm{G}$ Chemother 1980;17:757-61.

5. Kamimura T, Matsumoto Y, Okada N, Mine Y, Nishida M, Goto S, Kuwahara S. Ceftizoxime (FK 749), a new parenteral cephalosporin: in-vitro and in-vivo antibacterial activities. Antimicrob Agents Chemother 1979; 16:540-8.

6. Catlin BW. Iodometric detection of Haemophilus influenzaee beta-lactamase: rapid presumptive test for ampicillin resistance. Antimicrob Agents Chemother 1975; 7:265-70.

7. Kojo H, Nishida M, Goto S, Kuwahara S. Antibacterial $\stackrel{+}{-}$ activity of ceftizoxime (FK 749), a new cephalosporin, against $\square$ cephalosporin-resistant bacteria, and its stability to $\overline{0}$ $\beta$-lactamases. Antimicrob Agents Chemother 1979; 16:549-53.

8. Rajan VS, Sng EH, Pang R, Tan NJ, Thirumoorthy T, Yeo $\mathrm{KL}$. HR 756-a new cephalosporin in the treatment of $\frac{}{(\Phi}$ gonorrhoea caused by ordinary and penicillinase-producing 0 strains of Neisseria gonorrhoeae. $\mathrm{Br} J$ Vener Dis 1980; 56: 255-8. 